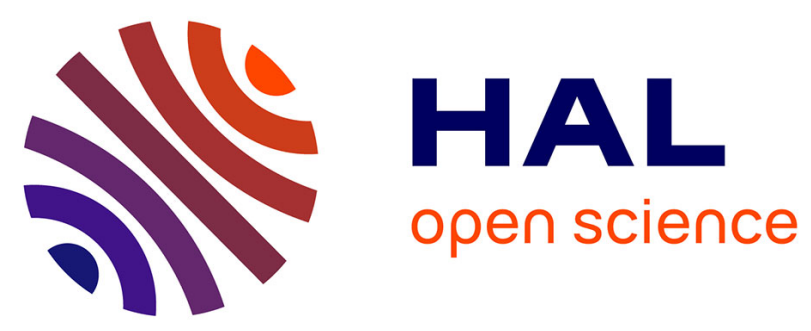

\title{
Sweet or Bland Dreams? Taste Loss in Isolated REM -Sleep Behavior Disorder and Parkinson's Disease
}

Milan Nigam, Ines Ayadi, Camille Noiray, Ana Branquino Bras, Erika Herraez

Sanchez, Smaranda Leu-Semenescu, Marie Vidailhet, Pauline Dodet, Isabelle Arnulf

\section{To cite this version:}

Milan Nigam, Ines Ayadi, Camille Noiray, Ana Branquino Bras, Erika Herraez Sanchez, et al.. Sweet or Bland Dreams? Taste Loss in Isolated REM -Sleep Behavior Disorder and Parkinson's Disease. Movement Disorders, 2021, 10.1002/mds.28692 . hal-03261753

\section{HAL Id: hal-03261753 https://hal.sorbonne-universite.fr/hal-03261753}

Submitted on 16 Jun 2021

HAL is a multi-disciplinary open access archive for the deposit and dissemination of scientific research documents, whether they are published or not. The documents may come from teaching and research institutions in France or abroad, or from public or private research centers.
L'archive ouverte pluridisciplinaire HAL, est destinée au dépôt et à la diffusion de documents scientifiques de niveau recherche, publiés ou non, émanant des établissements d'enseignement et de recherche français ou étrangers, des laboratoires publics ou privés. 


\section{Sweet or bland dreams? Taste loss in isolated REM-sleep behavior disorder and Parkinson's disease}

Milan Nigam, MD ${ }^{1,2,3}$, Ines Ayadi, MD¹, Camille Noiray, MD,1 Ana Branquino Bras, MD ${ }^{1,4}$, Erika Herraez Sanchez, MD1,5, Smaranda Leu-Semenescu, MD1, Marie Vidailhet, MD, PhD 6,7,8, Pauline Dodet, MD1, Isabelle Arnulf MD, $\mathrm{PhD} 1,7,8$

${ }^{1}$ Sleep Disorders Unit, University Hospital Pitié-Salpêtrière, APHP-Sorbonne, Paris, France

${ }^{2}$ Centre for Advanced Research in Sleep Medicine, Sacré-Coeur Hospital, Montreal, QC, Canada

${ }^{3}$ Department of Neurosciences, University of Montreal, Montreal, QC, Canada

${ }^{4}$ Department of Neurology - Centro Hospitalar e Universitário de Coimbra, Coimbra, Portugal

${ }^{5}$ Hospital Universitario La Paz, Madrid, Spain

${ }^{6}$ Department of Neurology, University Hospital Pitié-Salpêtrière, APHP-Sorbonne, Paris, France

${ }^{7}$ Institut du Cerveau et de la Moelle (Paris Brain Institute), Paris, France

${ }^{8}$ Sorbonne University, Paris, France

Keywords: REM sleep behavior disorder, Parkinson's disease, taste, hypogeusia

Institution at which the work was performed:

Sleep Disorders Unit, Pitié-Salpêtrière University Hospital, Paris, France

Financial support: The study was funded by the grant Icrin from ICM (Paris Brain Institute) to IA and benefited from the Iceberg project (Investissements d'Avenir, IAIHU-06 (Paris Institute of Neurosciences - IHU, soutien à la recherche contre la maladie de Parkinson Société Française de Médecine Esthétique, Monsieur Legrand; Energiepole Monsieur Mallart, Don de soutien a la recherche Monsieur Villain) to MV.

Manuscript submitted to: Movement disorders

All authors have seen and approved the manuscript

Disclosure of conflicts of interest:

MN has received an educational grant from Paladin Labs pharmaceuticals.

IAy has no conflicts of interest

PD has no conflicts of interest

SLS has speaking engagements with UCB Pharma.

IAr has received consultant fees from Idorsia Pharma, Ono Pharma, and speaker honoraria from UCB Pharma.

None of the financial disclosures is relevant to the submitted work.

Abstract word count: 142

Manuscript word count: 1695 words, 1 table and 1 figure, 4 supplemental tables

Running Title: taste in RBD

Corresponding author:

Dr. Isabelle Arnulf, MD, PhD 
Service des Pathologies du Sommeil, Hôpital Pitié-Salpêtrière

47-83 Bd de l'Hôpital, 75013 PARIS

Phone : 33 (0) 142167704

Fax : 33 (0) 142167700

E-mail: isabelle.arnulf@aphp.fr

\section{Abstract}

Background: Hyposmia and isolated REM sleep behavior disorder (iRBD) are well-established features of prodromal Parkinson's disease (PD).

Objectives: Evaluating whether taste loss (reported in PD and possibly suggesting brainstem involvement) is present at the iRBD stage.

Methods: We assessed taste function using the Taste Strip Test (evaluating 4 concentrations of bitter, sweet, sour and salty) in 44 participants with IRBD, 19 with PD and 29 controls. All participants underwent videopolysomnography, standardized questionnaires and clinical examination, including olfactory assessment.

Results: Participants with IRBD and PD had lower taste scores than controls. There was no difference between iRBD and PD cohorts nor was there any correlation between taste and olfaction, age, disease duration, cognition or autonomic function.

Conclusion: This study demonstrates for the first time the presence of taste impairment in iRBD that is independent of olfactory dysfunction and comparable to participants with PD.

\section{Introduction}

Hyposmia and isolated REM sleep behavior disorder (iRBD) are established features of prodromal Parkinson's disease (PD) and their co-occurrence has been shown to further increase patients' risk of conversion towards overt PD or other synucleinopathies. ${ }^{1}$ Hypogeusia is relatively understudied in PD compared with hyposmia. Previous studies have shown impaired taste in PD compared to controls. ${ }^{2-9}$ The timing and the mechanism hypogeusia has not been established and its prevalence in prodromal PD is uncertain, having never been studied in patients with iRBD. 
In this cross-sectional case-control study, we evaluate taste function in an RBD cohort, compared with age and sex matched controls and participants with PD, using a simple and validated test of gustatory function.

\section{Materials and Methods}

\section{Participants}

Controls, and participants with iRBD and PD were recruited between October 2019 and April 2021 among patients undergoing video-polysomnography (VPSG) or taking part in a larger RBD/PD cohort study at Pitié-Salpêtrière Hospital's Sleep Disorder Unit (Paris, France). IRBD was diagnosed as episodes of complex motor behavior occurring during REM sleep, or a history of enacted nightmares plus enhanced muscle tone during REM sleep in the absence of any other neurological disorder. ${ }^{10}$ Participants with PD were diagnosed by neurologists in accordance with MDS PD criteria. ${ }^{11}$ Controls were age and sex matched to the $\mathrm{RBDD}$ cohort and were selected among patients undergoing VPSG for indications other than parasomnias, with no previous history of neurological disease and in with no vPSG evidence of RBD. Participants were not eligible to participate in this trial if they had a history of Bell's palsy, otorhinolaryngological disorders or surgery affecting taste, or COVID-19. All participants gave written informed consent for participation in this study, in accordance with French research ethics laws.

\section{Procedures}

All participants underwent vPSG and a structured medical history assessing taste, smell, RBD symptoms and motor and non-motor features of PD. A complete neurological examination including the MDS-Unified Parkinson Disease Rating Scale part III (UPDRS-III) was performed. Taste was assessed using the Taste Strips Test (TST, Burghart Company, Germany). This validated tool employs 16 filter papers impregnated with four concentrations of four basic tastes (sweet, sour, salty and bitter). Strips are placed on the tongue, and participants are asked to identify the correct taste after moving the strip around with their mouth closed, rinsing the mouth with water before each new strip. One point is awarded for each correct answer for a maximum score of 16 . Pathological hypogeusia is defined by the manufacturer as a TST score less than 9. Olfaction was assessed using the Sniffin' Sticks Test (SST, 12 stick version, Burghart Company, Germany), which consists of 12 odour dispensing devices containing common odours 
that are identified from a four-item multiple choice list for a maximal score of 12. Cognition was assessed with the Montreal Cognitive Assessment (MoCA). Somnolence was assessed using Epworth Sleepiness Scale (ESS). Systolic orthostatic blood pressure drop was calculated from blood pressure measurements taken in the supine position and after one-minute standing.

\section{Statistical analysis}

Statistical significance was set below $P=.05$. Quantitative variables were compared using Kruskal-Wallis one-way analysis of variance with post-hoc testing for between-group significance using Wilcoxon signed rank test. Categorical variables were compared using Fisher's exact test, with Bonferroni adjustments for multiple testing. Spearman rank correlation was used to assess relationships between variables. All statistical analyses were computed using R (R (2020), R Foundation for Statistical Computing, Vienna, Austria).

\section{Results}

\section{Demographic and clinical characteristics of participants}

Table 1 summarizes the demographic and clinical characteristics of participants. A total of 44 participants with iRBD (37 men; mean age $68.4 \pm 9.0$; mean symptom duration: $6.1 \pm 3.5$ years), 19 participants with PD (11 men; mean age $72.2 \pm 7.2$; mean disease duration: $7.8 \pm 6.6$ years) and 29 controls (22 men; mean age $63.4 \pm 12.3$ ) were recruited. None of the participants with $\mathrm{RBD}$ have phenoconverted towards PD, multiple system atrophy or dementia with Lewy bodies during the study period. Fifteen (78.9\%) participants with PD also had RBD, three of whom had been diagnosed with iRBD prior to conversion to PD. Of the 29 controls, vPSG revealed obstructive sleep apnea $(\mathrm{N}=$ 18), restless leg syndrome $(N=3)$ and normal sleep $(N=8)$. Participants with PD were significantly older than controls, but not participants with iRBD. There were no differences in sex, smoking status or MoCA score between groups. Olfactory scores were weaker in participants with both $\mathrm{RBD}$ and PD when compared to controls but not when compared to each other. Autonomic dysfunction (higher systolic blood pressure drop and lower number of stools per week) was greater in participants with IRBD and PD compared to controls. ESS scores were higher in participants with PD than those with $\mathrm{RBD}$ and controls. 


\section{Assessment of taste}

Participants with PD were more likely to self-report hypogeusia (47.4\%) than participants with $\mathrm{RBD}(18.2 \%)$ and controls (0\%). Figure 1 shows the median and distributions of total TST scores across the three study groups. Participants with iRBD and PD scored lower on the TST when compared to controls. There was no further difference in TST scores between participants with iRBD and PD. The same pattern of taste loss in RRBD and PD was observed for each separate taste quality (in the 69 participants for whom taste sub-scores were available) and for all tastant concentrations (supplemental tables S1 and S2). The percentage of participants with a TST score below the cut-off for pathological hypogeusia of 9 was greater in participants with $\operatorname{RBD}(52.3 \%)$ and PD (63.1\%) compared to controls $(17.7 \%, P=.001)$.

Within both iRBD and PD cohorts, there were no significant correlations between participants' TST scores and their age, disease duration, olfactory function, orthostatic systolic blood pressure drop, bowel movement frequency or MDS-UPDRS III scores (supplemental tables S3 and S4).

\section{Discussion}

To our knowledge, this is the first study objectively demonstrating hypogeusia in participants with iRBD compared to controls. There was no significant difference in taste impairment between participants with iRBD and PD. This pattern of hypogeusia was observed for all individual tastes and tastant concentrations. We found no correlation between TST score and olfactory function, age, disease duration, autonomic or cognitive dysfunction.

Previously, a large community-based survey study demonstrated that self-reported hypogeusia is associated with a higher risk for probable iRBD.12 Our findings support this association, though in our study, participants with iRBD were not more likely than controls to self-report hypogeusia. Several cross-sectional studies employing varying measurement techniques (TST, Whole Mouth Testing, electrogustometry) have found that patients with PD more frequently have impaired taste when compared with controls. ${ }^{2-7,13}$ These studies have shown taste dysfunction to be independent of olfactory dysfunction (which is more frequently affected in both PD and iRBD) ${ }^{14}$, a finding reaffirmed in our study. Moreover, a large prospective case-control study in patients with idiopathic smell and/or taste loss has 
demonstrated that patients with combined smell and taste loss have an approximately threefold risk of developing PD compared to patients with hyposmia alone..$^{15}$

The mechanism of taste impairment in PD has not yet been established. According to Braak \& Braak's staging theory of PD, both first order (cranial nerves VII, IX and X) and second order taste neurons (nucleus of the solitary tract (NST)) are spared. ${ }^{16}$ This has led some authors to suggest that taste dysfunction may be related to cortical involvement of the primary gustatory cortices in advanced PD associated with dementia., 4,13 Indeed, a study assessing taste in PD dementia has revealed profound hypogeusia even when compared to other neurodegenerative dementias, whereas taste in non cognitively impaired patients with PD appears to be relatively mildly affected.,2,7

In contrast with the proposition that hypogeusia is a late feature of PD, cross-sectional studies have not demonstrated a correlation between PD duration and taste loss, $, 3,5$ and a prospective study assessing longitudinal evolution of hypogeusia in a cohort of 26 patients with PD showed no significant decline in taste over a mean followup period of 4.35 years. ${ }^{8}$ Furthermore, the ONSET-PD study showed that taste loss, when present, generally occurs 2-10 years before the onset of parkinsonian motor features. ${ }^{7}$ In our study, hypogeusia is similar in both iRBD and PD, suggesting that taste impairment begins early in the course of the disease, when the cortical burden of alphasynuclein is expected to be low. iRBD is related to pathology of the coeruleus/subcoeruleus complex and related brainstem structures, with relative sparing of the cortex. ${ }^{17} \mathrm{~A}$ recent neuropathological study has demonstrated that deposition of alpha-synuclein does occur in both the NST and cranial nerve nuclei in some patients with PD and Lewy body dementia, contrary to previous reports. ${ }^{18}$ The NST is directly adjacent to the dorsal motor nucleus of the vagus nerve (which is heavily involved in early PD) and murine models have shown monosynaptic connections between the nucleus of the solitary tract and the coeruleus/subcoeruleus complex. ${ }^{19-21}$ Thus, NST involvement could be consistent with transneuronal spread as per the prion-like theory of synucleinopathy pathogenesis. ${ }^{18}$ Collectively, these findings suggest that hypogeusia is an early, slowly progressive feature of PD which may be related to brainstem (rather than cortical) involvement. 
We acknowledge some limitations to our study. The smaller control and PD groups limit statistical power and increase the risk of sampling bias. The proportion of the PD group with RBD $(78.9 \%)$ is higher than the typically reported $46-59 \%{ }^{22-24}$ This is unlikely related to a referral bias, as only 4 participants with PD were referred specifically for a suspicion of RBD. It may reflect the fact that all of our participants underwent video-PSG, potentially leading to identification of milder forms of RBD that may have otherwise gone unrecognized. While this increases the comparability of the PD group to the RBD group, it may reduce the generalizability of our findings to patients with PD without RBD.

\title{
Conclusion
}

In conclusion, this study demonstrates taste impairment in participants with iRBD that is independent of olfactory dysfunction and comparable to PD. These findings support the hypothesis that hypogeusia is an early, slowly progressive feature of synucleinopathies related to brainstem involvement, rather than a late feature related to advanced cortical pathology. Further prospective investigations will be required to establish whether the identification of taste loss in RRD patients increases the risk of conversion to clinical PD or other synucleinopathies.

\author{
Abbeviations \\ iRBD: isolated REM sleep behavior disorder \\ PD: Parkinson's disease \\ vPSG: video-polysomnography \\ NST: nucleus of the solitary tract \\ TST: Taste Strip Test \\ SST: Sniffin' Sticks Test \\ ESS: Epworth sleepiness scale
}




\section{Author roles:}

Milan Nigam: Statistical analysis: Design and execution. Manuscript: Writing of the first draft.

Ines Ayadi: Control and patient recruitment and data collection

Camille Noiray: Patient recruitment and data collection

Ana Branquino Bras: Patient recruitment and data collection

Erika Herraez Sanchez: Patient recruitment and data collection

Pauline Dodet: Patient recruitment, review \& critique of manuscript.

Smaranda Leu-Semenescu: Patient recruitment, review \& critique of manuscript.

Marie Vidailhet: Founding, review \& critique of manuscript.

Isabelle Arnulf: Conception, founding and organization of research project. Review \& critique of manuscript and statistical analyses.

\section{Financial disclosures from the previous 12 months:}

Milan Nigam has received an educational grant from Paladin Labs pharmaceuticals

Ines Ayadi has nothing to disclose

Camille Noiray has nothing to disclose

Ana Branquino Bras has nothing to disclose

Erika Herraez Sanchez has nothing to disclose

Pauline Dodet has nothing to disclose

Smaranda Leu-Semenescu was paid speaker for UCB Pharma 
Marie Vidailhet received grants from Fondation d'Entreprise (EDF) and the Fondation Therese and Rene Planiol pour l'Etude du Cerveau.

Isabelle Arnulf has received consultancy honoraria from Idorsia Pharma and Ono Pharma.

\section{Ethical Compliance Statement}

The institutional local ethical standards committee approved the study. All participants gave written informed

consent. We confirm that we have read the Journal's position on issues involved in ethical publication and affirm that this work is consistent with those guidelines.

\section{References}

1. Postuma RB, Iranzo A, Hu M, et al. Risk and predictors of dementia and parkinsonism in idiopathic REM sleep behaviour disorder: a multicentre study. Brain. 2019;142(3):744-759.

2. Lang CJ, Leuschner T, Ulrich K, Stössel C, Heckmann JG, Hummel T. Taste in dementing diseases and parkinsonism. J Neurol Sci. 2006;248(1-2):177-184.

3. Shah M, Deeb J, Fernando M, et al. Abnormality of taste and smell in Parkinson's disease. Parkinsonism Relat Disord. 2009;15(3):232-237.

4. Deeb J, Shah M, Muhammed N, et al. A basic smell test is as sensitive as a dopamine transporter scan: comparison of olfaction, taste and DaTSCAN in the diagnosis of Parkinson's disease. QJM. 2010;103(12):941-952.

5. Kim HJ, Jeon BS, Lee JY, Cho YJ, Hong KS, Cho JY. Taste function in patients with Parkinson disease. J Neurol. 2011;258(6):1076-1079.

6. Cecchini MP, Osculati F, Ottaviani S, Boschi F, Fasano A, Tinazzi M. Taste performance in Parkinson's disease. J Neural Transm (Vienna). 2014;121(2):119-122.

7. Cecchini MP, Fasano A, Boschi F, Osculati F, Tinazzi M. Taste in Parkinson's disease. J Neurol. 2015;262(4):806-813.

8. Ricatti MJ, Ottaviani S, Boschi F, Fasano A, Tinazzi M, Cecchini MP. A prospective evaluation of taste in Parkinson's disease. J Neural Transm (Vienna). 2017;124(3):347-352.

9. Tarakad A, Jankovic J. Anosmia and Ageusia in Parkinson's Disease. Int Rev Neurobiol. 2017;133:541-556.

10. Sateia MJ. International classification of sleep disorders-third edition: highlights and modifications. Chest. 2014;146(5):1387-1394.

11. Postuma RB, Berg D, Stern M, et al. MDS clinical diagnostic criteria for Parkinson's disease. Mov Disord. 2015;30(12):1591-1601. 
12. Wong JC, Li J, Pavlova M, et al. Risk factors for probable REM sleep behavior disorder: A community-based study. Neurology. 2016;86(14):1306-1312.

13. Oppo V, Melis M, Melis M, Tomassini Barbarossa I, Cossu G. "Smelling and Tasting" Parkinson's Disease: Using Senses to Improve the Knowledge of the Disease. Front Aging Neurosci. 2020;12:43.

14. Postuma RB, Gagnon JF, Vendette M, Montplaisir JY. Markers of neurodegeneration in idiopathic rapid eye movement sleep behaviour disorder and Parkinson's disease. Brain. 2009;132(12):3298-3307.

15. Haehner A, Masala C, Walter S, Reichmann H, Hummel T. Incidence of Parkinson's disease in a large patient cohort with idiopathic smell and taste loss. J Neurol. 2019;266(2):339-345.

16. Braak H, Del Tredici K, Bratzke H, Hamm-Clement J, Sandmann-Keil D, Rüb U. Staging of the intracerebral inclusion body pathology associated with idiopathic Parkinson's disease (preclinical and clinical stages). J Neurol. 2002;249 Suppl 3:III/1-5.

17. García-Lorenzo D, Longo-Dos Santos C, Ewenczyk C, et al. The coeruleus/subcoeruleus complex in rapid eye movement sleep behaviour disorders in Parkinson's disease. Brain. 2013;136(Pt 7):2120-2129.

18. Seidel K, Mahlke J, Siswanto S, et al. The brainstem pathologies of Parkinson's disease and dementia with Lewy bodies. Brain Pathol. 2015;25(2):121-135.

19. Van Bockstaele EJ, Peoples J, Telegan P. Efferent projections of the nucleus of the solitary tract to peri-locus coeruleus dendrites in rat brain: evidence for a monosynaptic pathway. J Comp Neurol. 1999;412(3):410-428.

20. Lopes LT, Patrone LG, Li KY, et al. Anatomical and functional connections between the locus coeruleus and the nucleus tractus solitarius in neonatal rats. Neuroscience. 2016;324:446-468.

21. Kawai Y. Differential Ascending Projections From the Male Rat Caudal Nucleus of the Tractus Solitarius: An Interface Between Local Microcircuits and Global Macrocircuits. Frontiers in Neuroanatomy. 2018;12(63).

22. De Cock VC, Vidailhet M, Leu S, et al. Restoration of normal motor control in Parkinson's disease during REM sleep. Brain. 2007;130(Pt 2):450-456.

23. Postuma RB, Gagnon JF, Vendette M, Charland K, Montplaisir J. REM sleep behaviour disorder in Parkinson's disease is associated with specific motor features. $J$ Neurol Neurosurg Psychiatry. 2008;79(10):1117-1121.

24. Sixel-Döring F, Trautmann E, Mollenhauer B, Trenkwalder C. Associated factors for REM sleep behavior disorder in Parkinson disease. Neurology. 2011;77(11):1048-1054. 


\section{Figure legend:}

Figure 1: Taste Strip Test Score (out of 16) in controls, participants with idiopathic REM sleep behavior disorder (iRBD) and Parkinson's disease (PD). Median value (thick horizontal bar) and first and third quartiles (superimposed box plot). 
Table S1: Taste Strip Test sub-scores for individual tastes in controls, participants with idiopathic REM sleep behavior disorder (iRBD) and with Parkinson's disease (PD)

\begin{tabular}{|c|c|c|c|c|c|}
\hline & $\begin{array}{l}\text { Controls } \\
(n=29), A\end{array}$ & $\begin{array}{c}\text { iRBD } \\
(n=26), B\end{array}$ & $\begin{array}{c}\text { PD } \\
(14), C\end{array}$ & $P$ & $\begin{array}{c}\text { Post hoc } \\
\text { significance }\end{array}$ \\
\hline Age & $64.0(12.4)$ & $70.0(9.2)$ & $71.8(7.0)$ & .06 & - \\
\hline Male, n (\%) & $22(75.9)$ & $22(84.6)$ & $8(57.1)$ & .2 & - \\
\hline $\begin{array}{l}\text { Current } \\
\text { smokers, n (\%) }\end{array}$ & $3(10.3)$ & $4(15.3)$ & $0(0)$ & .4 & - \\
\hline \multicolumn{6}{|c|}{ TST sub-score by taste } \\
\hline Sweet & $3.5(0.6)$ & $2.6(1.3)$ & $2.0(1.4)$ & $<.001$ & $\mathrm{~A}>\mathrm{B}^{* *}, \mathrm{C}^{* * *}$ \\
\hline Bitter & $3.0(1.2)$ & $1.0(1.2)$ & $1.3(1.3)$ & $<.001$ & $A>B^{* * *}, C^{* * *}$ \\
\hline Salty & $3.1(0.9)$ & $1.7(0.9)$ & $1.7(1.1)$ & $<.001$ & $\mathrm{~A}>\mathrm{B} * * *, \mathrm{C}^{* * *}$ \\
\hline Sour & $2.8(1.1)$ & $1.7(1.0)$ & $1.4(1.0)$ & $<.001$ & $\mathrm{~A}>\mathrm{B}^{* *}, \mathrm{C}^{* *}$ \\
\hline \multicolumn{6}{|c|}{ TST sub-scores below pathological cut-off ${ }^{\dagger}, \mathrm{n}(\%)$} \\
\hline Sweet & $0(0)$ & $7(24.1)$ & $6(20.7)$ & $<.001$ & $\mathrm{~A}>\mathrm{B}^{* *}, \mathrm{C}^{* * *}$ \\
\hline Bitter & $1(3.4)$ & $12(41.4)$ & $5(17.2)$ & $<.001$ & $A>B^{* * *}, C^{*}$ \\
\hline Salty & $1(3.4)$ & $10(34.5)$ & $5(17.2)$ & .002 & $A>B^{* *}, C^{*}$ \\
\hline Sour & $5(17.2)$ & $12(41.4)$ & $7(24.1)$ & .03 & $A>B^{*}, C^{*}$ \\
\hline
\end{tabular}

Results are presented as means \pm standard deviation unless otherwise indicated.

+ TST sub-scores $<2$ are pathological, except bitter which requires a score $<1$

${ }^{*} P<0.05,{ }^{* *} P<0.01,{ }^{* * *} P<0.001$ 
Table S2: Taste Strip Test sub-scores for tastant concentration strength in controls, participants with idiopathic REM sleep behavior disorder (iRBD) and with Parkinson's disease (PD)

\begin{tabular}{lllllc}
\multicolumn{2}{c}{ Controls (29) } & iRBD (44) & PD (19) & p & Post hoc \\
\hline \multicolumn{2}{l}{ TST sub-score by tastant concentration } \\
\hline Very weak & $2.0(1.3)$ & $1.2(1.1)$ & $0.9(1.6)$ & .006 & $\mathrm{~A}>\mathrm{B}^{*}, \mathrm{C}^{*}$ \\
\hline Weak & $3.0(0.9)$ & $1.7(1.2)$ & $1.6(1.4)$ & $<.001$ & $\mathrm{~A}>\mathrm{B}^{* * *}, \mathrm{C}^{* *}$ \\
\hline Strong & $3.5(0.8)$ & $2.2(1.1)$ & $2.2(1.5)$ & $<.001$ & $\mathrm{~A}>\mathrm{B}^{* * *}, \mathrm{C}^{* *}$ \\
\hline Very strong & $3.8(0.6)$ & $3.0(1.1)$ & $2.8(1.2)$ & .001 & $\mathrm{~A}>\mathrm{B}^{* *}, \mathrm{C}^{* *}$ \\
\hline
\end{tabular}

Results are presented as means \pm standard

${ }^{*} P<0.05,{ }^{* *} P<0.01,{ }^{* * *} P<0.001$ 
Table S3: Correlation between scores at the Taste Strip Test and other clinical measures in participants with iRBD

\begin{tabular}{lcc} 
& Correlation coefficient (rho) & $p$-value \\
\hline Age & -0.2 & 0.1 \\
Duration of RBD symptoms & -0.1 & 0.9 \\
Olfaction (SST score) & -0.2 & 0.3 \\
Stools per week & 0.01 & 0.9 \\
Systolic blood pressure drop & 0.2 & 0.4 \\
UPDRS III & -0.1 & 0.7 \\
Montreal Cognitive Assessment & 0.1 & 0.6 \\
Epworth sleepiness scale & 0.02 & 0.9
\end{tabular}

SST: Sniffin' Sticks Test; UPDRS-III: Unified Parkinson's Disease Rating Scale - part 3. 
Table S4: Correlation between scores at the Taste Strip Test and other clinical measures in participants with PD

Correlation coefficient (rho)

\begin{tabular}{lc}
\hline Age & 0.2 \\
Time since PD diagnosis & -0.1 \\
Olfaction (SST score) & 0.3 \\
Stools per week & -0.2 \\
Systolic blood pressure drop & -0.2 \\
UPDRS III & 0.4 \\
Montreal Cognitive Assessment & 0.4 \\
Epworth sleepiness scale & -0.1 \\
SST: Sniffin' Sticks Test; UPDRS-III: Unified Parkinson's Disease Rating Scale - part 3.
\end{tabular}

$p$-value

0.5

0.8

0.3

0.5

0.6

0.2

0.3

0.9 


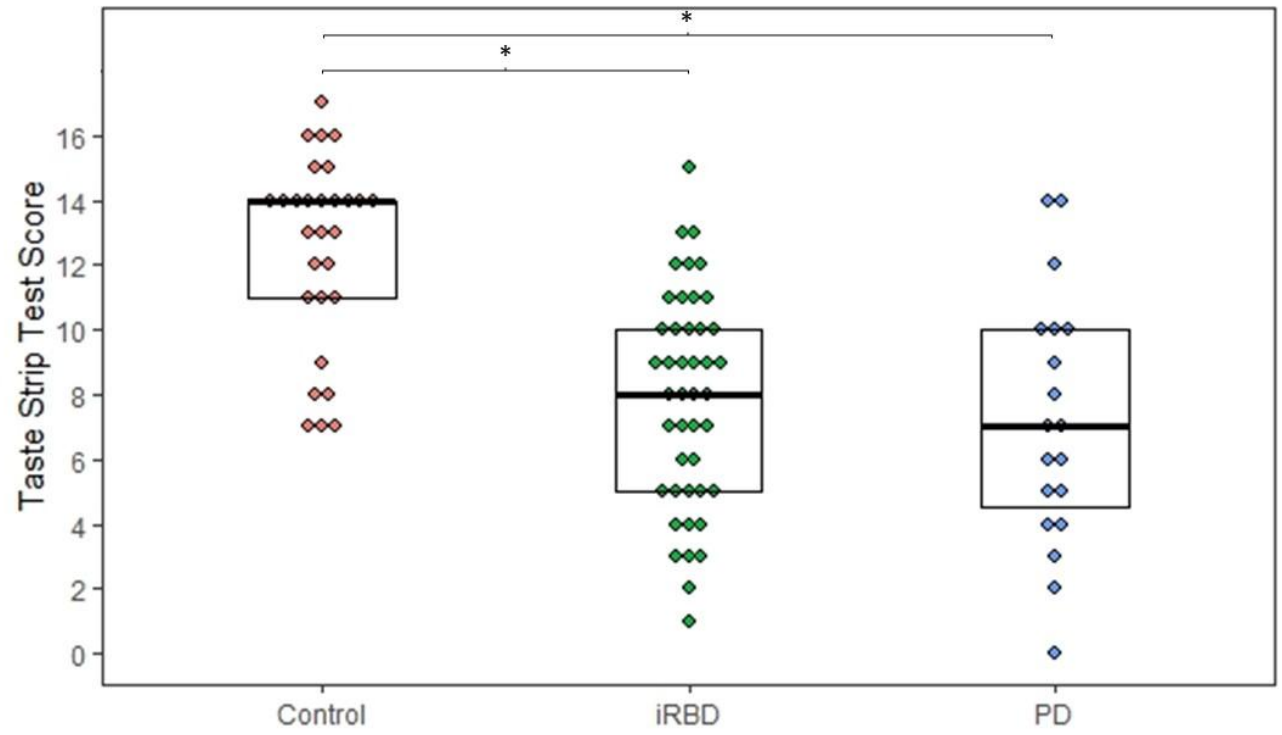

Figure 1: Taste Strip Test Score (out of 16) in controls, participants with idiopathic REM sleep behavior disorder (iRBD) and Parkinson's disease (PD). Median value (thick horizontal bar) and first and third quartiles (superimposed box plot). 